PHYSICAL REVIEW D 90, 012010 (2014)

\title{
Observation of muon intensity variations by season with the MINOS near detector
}

P. Adamson, ${ }^{7}$ I. Anghel, ${ }^{14,1}$ A. Aurisano, ${ }^{6}$ G. Barr, ${ }^{20}$ M. Bishai, ${ }^{2}$ A. Blake, ${ }^{4}$ G. J. Bock, ${ }^{7}$ D. Bogert, ${ }^{7}$ S. V. Cao, ${ }^{28}$ C. M. Castromonte, ${ }^{8}$ S. Childress, ${ }^{7}$ J. A. B. Coelho, ${ }^{29,5}$ L. Corwin, ${ }^{13}$ D. Cronin-Hennessy, ${ }^{17}$ J. K. de Jong, ${ }^{20}$ A. V. Devan, ${ }^{31}$ N. E. Devenish, ${ }^{26}$ M. V. Diwan, ${ }^{2}$ C. O. Escobar, ${ }^{5}$ J. J. Evans,${ }^{16}$ E. Falk ${ }^{26}$ G. J. Feldman, ${ }^{9}$ T. H. Fields, ${ }^{1}$ M. V. Frohne, ${ }^{10}$ H. R. Gallagher, ${ }^{29}$ R. A. Gomes, ${ }^{8}$ M. C. Goodman, ${ }^{1}$ P. Gouffon, ${ }^{23}$ N. Graf, ${ }^{12}$ R. Gran, ${ }^{18}$ K. Grzelak, ${ }^{30}$ A. Habig, ${ }^{18}$ S. R. Hahn ${ }^{7}$ J. Hartnell, ${ }^{26}$ R. Hatcher, ${ }^{7}$ A. Holin, ${ }^{15}$ J. Huang, ${ }^{28}$ J. Hylen, ${ }^{7}$ G. M. Irwin, ${ }^{25}$ Z. Isvan, ${ }^{2,21}$ C. James, ${ }^{7}$ D. Jensen, ${ }^{7}$ T. Kafka, ${ }^{29}$ S. M. S. Kasahara, ${ }^{17}$ G. Koizumi, ${ }^{7}$ M. Kordosky, ${ }^{31}$ A. Kreymer, ${ }^{7}$ K. Lang, ${ }^{28}$ J. Ling, ${ }^{2}$ P. J. Litchfield, ${ }^{17,22}$ P. Lucas, ${ }^{7}$ W. A. Mann, ${ }^{29}$ M. L. Marshak,${ }^{17}$ M. Mathis, ${ }^{31}$ N. Mayer, ${ }^{29,13}$ C. McGivern,${ }^{21}$ M. M. Medeiros,${ }^{8}$ R. Mehdiyev, ${ }^{28}$ J. R. Meier, ${ }^{17}$ M. D. Messier, ${ }^{13}$ W. H. Miller, ${ }^{17}$ S. R. Mishra, ${ }^{24}$ S. Moed Sher, ${ }^{7}$ C. D. Moore, ${ }^{7}$ L. Mualem, ${ }^{3}$ J. Musser, ${ }^{13}$ D. Naples, ${ }^{21}$ J. K. Nelson, ${ }^{31}$ H. B. Newman, ${ }^{3}$ R. J. Nichol, ${ }^{15}$ J. A. Nowak, ${ }^{17}$ J. O’Connor, ${ }^{15}$ M. Orchanian, ${ }^{3}$ S. Osprey, ${ }^{20}$ R. B. Pahlka, ${ }^{7}$ J. Paley, ${ }^{1}$ R. B. Patterson, ${ }^{3}$ G. Pawloski,${ }^{17,25}$ A. Perch, ${ }^{15}$ S. Phan-Budd, ${ }^{1}$ R. K. Plunkett, ${ }^{7}$ N. Poonthottathil ${ }^{7}$ X. Qiu ${ }^{25}$ A. Radovic, ${ }^{15}$ B. Rebel, ${ }^{7}$ C. Rosenfeldd ${ }^{24}$ H. A. Rubin, ${ }^{12}$ M. C. Sanchez, ${ }^{14,1}$ J. Schneps, ${ }^{29}$ A. Schreckenberger, ${ }^{17}$ P. Schreiner, ${ }^{1}$ R. Sharma, ${ }^{7}$ A. Sousa, ${ }^{6,9}$ N. Tagg, ${ }^{19}$ R. L. Talaga, ${ }^{1}$ J. Thomas, ${ }^{15}$ M. A. Thomson, ${ }^{4}$ X. Tian, ${ }^{24}$ A. Timmons,${ }^{16}$ S. C. Tognini, ${ }^{8}$ R. Toner, ${ }^{9,4}$ D. Torretta, ${ }^{7}$ J. Urheim, ${ }^{13}$ P. Vahle, ${ }^{31}$ B. Viren, ${ }^{2}$ A. Weber, ${ }^{20,22}$ R. C. Webb,${ }^{27}$ C. White, ${ }^{12}$ L. Whitehead, ${ }^{11,2}$ L. H. Whitehead, ${ }^{15}$ S. G. Wojcicki, ${ }^{25}$ and R. Zwaska ${ }^{7}$

(MINOS Collaboration)

\author{
${ }^{1}$ Argonne National Laboratory, Argonne, Illinois 60439, USA \\ ${ }^{2}$ Brookhaven National Laboratory, Upton, New York 11973, USA \\ ${ }^{3}$ Lauritsen Laboratory, California Institute of Technology, Pasadena, California 91125, USA \\ ${ }^{4}$ Cavendish Laboratory, University of Cambridge, \\ Madingley Road, Cambridge CB3 OHE, United Kingdom \\ ${ }^{5}$ Universidade Estadual de Campinas, IFGW-UNICAMP, C.P. 6165, Campinas, \\ 13083-970 São Paulo, Brazil \\ ${ }^{6}$ Department of Physics, University of Cincinnati, Cincinnati, Ohio 45221, USA \\ ${ }^{7}$ Fermi National Accelerator Laboratory, Batavia, Illinois 60510, USA \\ ${ }^{8}$ Instituto de Física, Universidade Federal de Goiás, C.P. 131, Goiânia 74001-970, Brazil \\ ${ }^{9}$ Department of Physics, Harvard University, Cambridge, Massachusetts 02138, USA \\ ${ }^{10}$ Holy Cross College, Notre Dame, Indiana 46556, USA \\ ${ }^{11}$ Department of Physics, University of Houston, Houston, Texas 77204, USA \\ ${ }^{12}$ Department of Physics, Illinois Institute of Technology, Chicago, Illinois 60616, USA \\ ${ }^{13}$ Indiana University, Bloomington, Indiana 47405, USA \\ ${ }^{14}$ Department of Physics and Astronomy, Iowa State University, Ames, Iowa 50011, USA \\ ${ }^{15}$ Department of Physics and Astronomy, University College London, Gower Street, \\ London WC1E 6BT, United Kingdom \\ ${ }^{16}$ School of Physics and Astronomy, University of Manchester, Oxford Road, \\ Manchester M13 9PL, United Kingdom \\ ${ }^{17}$ University of Minnesota, Minneapolis, Minnesota 55455, USA \\ ${ }^{18}$ Department of Physics, University of Minnesota Duluth, Duluth, Minnesota 55812, USA \\ ${ }^{19}$ Otterbein College, Westerville, Ohio 43081, USA \\ ${ }^{20}$ Subdepartment of Particle Physics, University of Oxford, Oxford OX1 3RH, \\ United Kingdom \\ ${ }^{21}$ Department of Physics and Astronomy, University of Pittsburgh, Pittsburgh, \\ Pennsylvania 15260, USA \\ ${ }^{22}$ Rutherford Appleton Laboratory, Science and Technologies Facilities Council, Didcot OX11 OQX, \\ United Kingdom \\ ${ }^{23}$ Instituto de Física, Universidade de São Paulo, C.P. 66318, São Paulo 05315-970, Brazil \\ ${ }^{24}$ Department of Physics and Astronomy, University of South Carolina, \\ Columbia, South Carolina 29208, USA \\ ${ }^{25}$ Department of Physics, Stanford University, Stanford, California 94305, USA \\ ${ }^{26}$ Department of Physics and Astronomy, University of Sussex, Falmer, \\ Brighton BN1 9QH, United Kingdom \\ ${ }^{27}$ Physics Department, Texas A\&M University, College Station, Texas 77843, USA \\ ${ }^{28}$ Department of Physics, University of Texas at Austin, \\ 1 University Station C1600, Austin, Texas 78712, USA \\ ${ }^{29}$ Physics Department, Tufts University, Medford, Massachusetts 02155, USA
}


${ }^{30}$ Department of Physics, University of Warsaw, Hoża 69, PL-00-681 Warsaw, Poland

${ }^{31}$ Department of Physics, College of William \& Mary, Williamsburg, Virginia 23187, USA

(Received 20 June 2014; published 22 July 2014)

A sample of $1.53 \times 10^{9}$ cosmic-ray-induced single muon events has been recorded at $225 \mathrm{~m}$ water equivalent using the MINOS near detector. The underground muon rate is observed to be highly correlated with the effective atmospheric temperature. The coefficient $\alpha_{T}$, relating the change in the muon rate to the change in the vertical effective temperature, is determined to be $0.428 \pm 0.003$ (stat.) \pm 0.059 (syst.). An alternative description is provided by the weighted effective temperature, introduced to account for the differences in the temperature profile and muon flux as a function of zenith angle. Using the latter estimation of temperature, the coefficient is determined to be $0.352 \pm 0.003$ (stat.) \pm 0.046 (syst.).

\section{INTRODUCTION}

It is well known that the fluxes of cosmic ray muons observed in underground detectors exhibit a seasonal variation. The flux variations are attributed to density variations in the atmosphere, where mesons from primary cosmic ray interactions are themselves strongly interacting or decaying via the weak interaction. During the summer, increases in the temperature cause the atmosphere to expand, reducing the probability that a secondary meson will interact. Consequently, the muon flux from weak meson decays increases. This variation in the muon flux has been observed, and correlated with temperature changes, by a number of experiments [1-11], including the MINOS measurement at the far detector (FD) [12]. These experiments measure $\alpha_{T}$, the correlation coefficient between the muon flux and the atmospheric temperature. This coefficient varies as a function of overburden. The much shallower MINOS near detector (ND), with 225 mwe overburden, is at a depth where $\alpha_{T}$ is expected to be rapidly changing as a function of overburden and has never before been accurately measured. In this paper, we make the first measurement of $\alpha_{T}$ in this important region. We also develop a novel formalism that takes into account the variation in atmospheric overburden, and hence the effective temperature, as a function of zenith angle.

Meson decays take place over a range of altitudes where the temperature is changing. It is customary to define an effective atmospheric temperature $T_{\text {eff }}$, described in Sec. II $\mathrm{B}$, where the muons originate. The variation in the observed muon rate $R_{\mu}$ compared to the mean rate $\left\langle R_{\mu}\right\rangle$ can be expressed in terms of a similar change in $T_{\text {eff }}$ :

$$
\frac{\Delta R_{\mu}}{\left\langle R_{\mu}\right\rangle}=\alpha_{T} \frac{\Delta T_{\text {eff }}}{\left\langle T_{\text {eff }}\right\rangle},
$$

where $\left\langle R_{\mu}\right\rangle$ is equivalent to the rate for an effective atmospheric temperature $\left\langle T_{\text {eff }}\right\rangle$. The magnitude of the temperature coefficient $\alpha_{T}$ depends upon the muon energy and hence upon the depth of the detector. The parameter $\alpha_{T}$ is larger for detectors situated deeper underground because the muons originate from higher energy mesons which have increased lifetimes due to time dilation. This parameter is reduced for shallow detectors because as the atmospheric temperature increases the primary interaction occurs at a higher altitude, increasing the probability that the muon will itself decay prior to reaching the detector. A measurement of the temperature coefficient may be used to measure the $\mathrm{K} / \pi$ ratio at energies beyond the reach of current fixed target experiments [13]. Moreover the size of the detector, combined with its angular resolution, has allowed the first measurements of $\alpha_{T}$ as a function of the muon zenith angle. The data analyzed in this work consist of single muon events recorded by the MINOS ND over the six-year period between June 1, 2006 and April 30, 2012.

The selection of the experimental data sets is presented in Sec. II below. Section III presents the measurement of the temperature coefficient $\alpha_{T}$ along with the theoretical prediction. In Sec. IV the dependence of $\alpha_{T}$ on the muon zenith angle is examined. The observations motivate the introduction of a new formula for the effective temperature which improves upon the approximations that are present in the standard analysis. Conclusions from the analysis of this work are presented in Sec. V.

\section{THE DATA SETS}

The measurement of the temperature coefficient $\alpha_{T}$ has been performed using muon data collected by the MINOS ND and temperature data provided by the European Center for Medium-Range Weather Forecasts (ECMWF) [14].

\section{A. MINOS near detector muon data}

The 0.98 kton MINOS ND [15] is a magnetized steel and scintillator sampling calorimeter designed to measure neutrino interactions in the Fermilab NuMI beam [16]. It is located at Fermilab. The detector, whose dimensions are $3.8 \mathrm{~m}$ height $\times 4.8 \mathrm{~m}$ width $\times 16.6 \mathrm{~m}$ length, contains 282 vertical planes. Each of the first 120 planes consists of a $2.54 \mathrm{~cm}$ thick steel plane, a $1 \mathrm{~cm}$ thick scintillator layer and a small air gap. The scintillator layers are composed of either 64 or 96 scintillating strips, each $4.1 \mathrm{~cm}$ wide. In the 


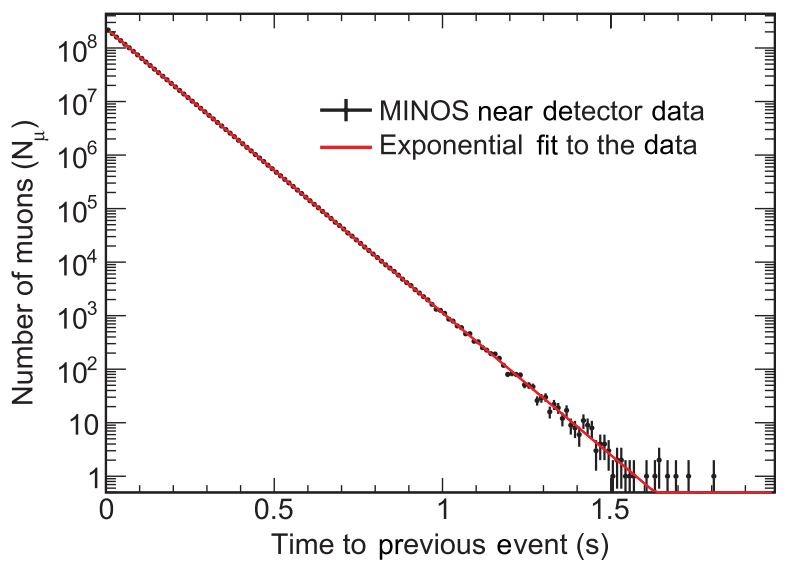

FIG. 1 (color online). Time between neighboring single atmospheric muon events in the MINOS ND. The data is well fitted to an exponential distribution with a mean rate $12.2374 \pm 0.0003 \mathrm{~Hz}$.

latter 162 planes only one in five steel planes have an attached scintillating layer. The strips in neighboring planes are orthogonal to allow for three-dimensional track reconstruction. The scintillating strips are read out by 64-pixel multianode photomultiplier tubes (PMT) [17].

Each PMT pixel is digitized continuously at $53.1 \mathrm{MHz}$ (18.83 ns). For this analysis, a cosmic trigger was used [15]; the trigger was produced when either four strips in five sequential planes, or when strips from any 20 planes, registered a signal above the $1 / 3$ photoelectron dynode threshold within $151 \mathrm{~ns}$. This trigger rate at the MINOS ND is approximately $27 \mathrm{~Hz}$.

The atmospheric muon selection applied to the cosmic trigger data requires that the event contains one wellreconstructed downward-going track collected during a period of good detector running conditions. The requirements are the same as those used for the MINOS analysis of the ND charge ratio [18] up to the charge sign quality selection in that analysis. Comparison with Monte Carlo shows that backgrounds and misreconstruction errors are negligible. Figure 1 demonstrates that the distribution of the time between consecutive muon events is exponential, as expected. In total over $1.53 \times 10^{9}$ single muon events have been selected with a mean rate of $12.2374 \pm$ $0.0003 \mathrm{~Hz}$. The trigger rate above reflects real muons, and the reduction is mostly due to the fact that the scintillator coverage in the ND is smaller than the steel. This geometry effect has no impact on the seasonal variation.

\section{B. Effective temperature}

The temperature as a function of atmospheric depth has been determined using the ECMWF atmospheric model [14]. The ECMWF procedure collates a number of different types of observations (e.g., surface, satellite and upper air sounding) at approximately 640 locations around the globe; the data are contiguous both spatially and in time.
The ECMWF global atmospheric model interpolates to a particular location assuming a smooth function of one degree latitude and longitude bins, and in varying elevation bins. For the MINOS ND at Fermilab, the model calculates atmospheric temperatures at 37 different, unevenly spaced pressure levels between $1 \mathrm{hPa}$ and $1000 \mathrm{hPa}$ at four times throughout the 24 hour day $(0000 \mathrm{~h}, 0600 \mathrm{~h}, 1200 \mathrm{~h}$ and $1800 \mathrm{~h}$ ). An earlier version of the ECMWF model calculates temperatures at 21 pressure levels, and was used to help determine the sensitivity of the $\alpha_{T}$ fits. By comparing the ECMWF temperature data with that of the Integrated Global Radiosonde Archive [19], it was determined that the uncertainties are $0.31 \mathrm{~K}$. As is reported in Ref. [12], the systematic uncertainty for this temperature model is estimated to be $0.2 \%$.

The lack of data above a height corresponding to $1 \mathrm{hPa}$ does not affect the results of this analysis as the depth $X$ of the atmosphere above $1 \mathrm{hPa}\left(1 \mathrm{hPa}=1.019 \mathrm{~g} / \mathrm{cm}^{2}\right)$ is insufficient to produce a statistically significant number of muons. Since it is not possible to determine where in the atmosphere a particular muon originated, a single effective temperature is defined [13,20], $T_{\text {eff }}$, which is the weighted $W(X)$ average based on the expected muon production spectrum

$$
T_{\text {eff }}=\frac{\int_{0}^{\infty} d X T(X) W(X)}{\int_{0}^{\infty} d X W(X)}
$$

Since the temperature $T(X)$ is measured at 37 discrete depths, a numerical integration is performed based on a quadratic interpolation between temperature measurements. The atmospheric depth depends on both $\pi$ and $\mathrm{K}$ decay, so $W(X)=W^{\pi}(X)+W^{K}(X)$. Figure 2 shows the mean temperatures, averaged over the analysis period,

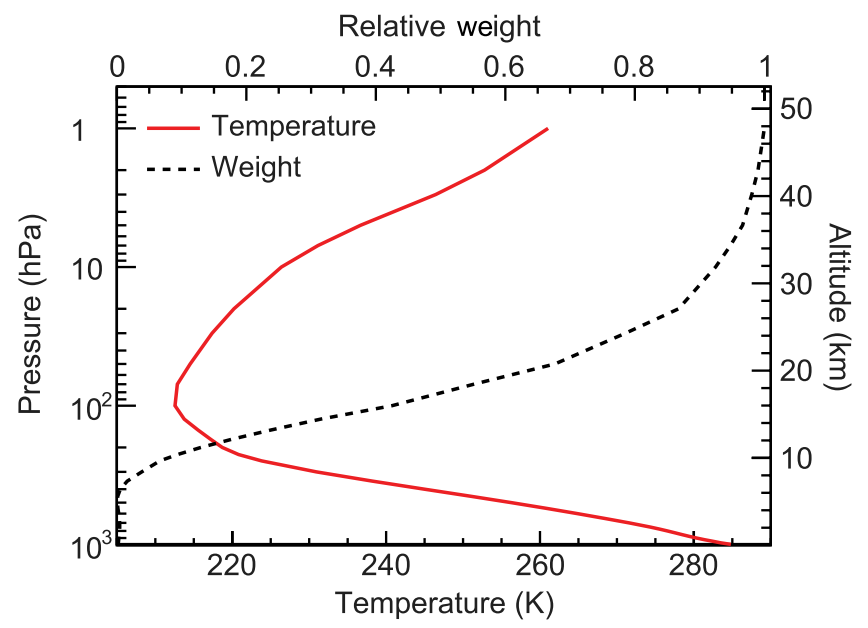

FIG. 2 (color online). The average temperature (solid red line) [14] and normalized weights $W(X)$ (blacked dashed line) as a function of pressure level at the MINOS ND site. The right vertical axis shows the altitude corresponding to a particular pressure. 
and the normalized weight $W(X)$ as a function of atmospheric depth.

These weights are

$$
W^{\pi(K)}(X) \approx \frac{\left(1-X / \Lambda_{\pi(K)}^{\prime}\right)^{2} e^{-X / \Lambda_{\pi(K)}} A_{\pi(K)}^{1}}{\gamma+(\gamma+1) B_{\pi(K)}^{1} K(X)\left(\frac{\left\langle E_{\mathrm{th}} \cos \theta\right\rangle}{\epsilon_{\pi(K)}}\right)^{2}}
$$

where

$$
K(X)=\frac{\left(1-X / \Lambda_{\pi(K)}^{\prime}\right)^{2}}{\left(1-e^{-X / \Lambda_{\pi(K)}^{\prime}}\right) \Lambda_{\pi(K)}^{\prime} / X} .
$$

The attenuation lengths of the cosmic ray primary, pion and kaon are $\Lambda_{N}, \Lambda_{\pi}$ and $\Lambda_{K}$ respectively. $\Lambda_{\pi(K)}^{\prime}$ is defined as $1 / \Lambda_{\pi(K)}^{\prime}=1 / \Lambda_{N}-1 / \Lambda_{\pi(K)}$. The parameters $A_{\pi(K)}^{1}$ account for inclusive meson production in the forward fragmentation region, the masses of mesons and muons and the muon spectral index $\gamma[13,20]$. The parameters $B_{\pi(K)}^{1}$ reflect the relative attenuation of mesons in the atmosphere. The critical energy of the mesons $\epsilon_{\pi(K)}$ is the energy at which the probability of meson decay and interaction are equal. $E_{\mathrm{th}}$ is the minimum energy required for a muon to survive to a particular depth and $\theta$ is the zenith angle of the muon. Apart from the value of $\left\langle E_{\mathrm{th}} \cos \theta\right\rangle$, which has a mean value of $54 \mathrm{GeV}$ at the MINOS ND, the values used for the parameters in Eqs. (3) and (4) are the same as in Refs. $[8,12]$.

\section{DATA ANALYSIS}

Equation (1) states that the change in the observed muon rate is related to the change in the effective atmospheric temperature. In this section we will present the MINOS ND muon and ECMWF temperature data as a function of time. The value of $\alpha_{T}$ is then determined by comparing the effective temperature determined from a single ECMWF temperature measurement to the corresponding six hours of MINOS muon data ( \pm 3 hours on either side). The effect of surface pressure on the muon rate was investigated and found to be small $[21,22]$. It had no impact on the measurement of $\alpha_{T}$ and is therefore not considered further.

\section{A. Seasonal variations}

Figure 3 displays the effective atmospheric temperature, as defined by Eq. (2), directly above the MINOS ND as a function of time. Figure 4 shows the observed muon rate at the MINOS ND as a function of time. The gaps in the data correspond to periods when the ND was not running or when the detector failed the data quality criteria.

Both the MINOS ND muon and effective temperature data have clear modulation signatures. The nominal modulation parameters were determined by fitting the data to an equation of the form

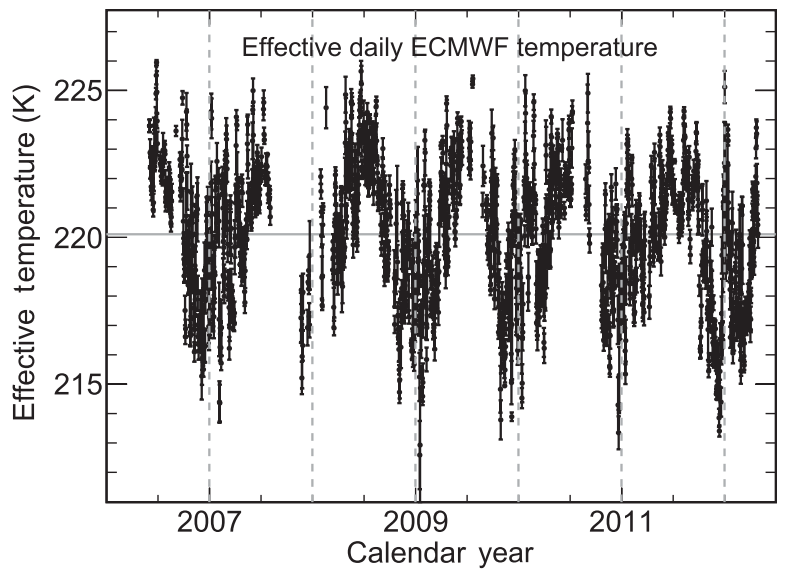

FIG. 3. Effective temperature as a function of time for the atmosphere directly above the MINOS ND. Each data point corresponds to one day of ECMWF data. The mean value is the average of the four ECMWF data points for that day. The $y$-axis errors are the standard deviation of those points. The solid horizontal line is the mean effective temperature $\left\langle T_{\text {eff }}\right\rangle=220.1 \mathrm{~K}$. The dashed vertical lines denote the start of new calendar years.

$$
R(t)=R_{0}\left(1+A \cdot \cos \left[\frac{2 \pi}{P}\left(t-t_{0}\right)\right]\right),
$$

where $R_{0}$ is mean value, $A$ is the fractional modulation amplitude and $P$ is the period. The time $t$ is the number of days elapsed since January 1,2010 . The phase $t_{0}$ is the first day at which the signal is at a maximum. Fitting the MINOS ND muon data in Fig. 4 to Eq. (5) yields a mean rate of $12.2458 \pm 0.0003 \mathrm{~Hz}$, a period of $367.8 \pm 0.4$ days and a phase of $200.9 \pm 0.8$ days. Fitting the effective temperature data in Fig. 3 to Eq. (5) yields a mean value of $220.1 \pm 0.2 \mathrm{~K}$, a period of $365.0 \pm 0.1$ days and a phase

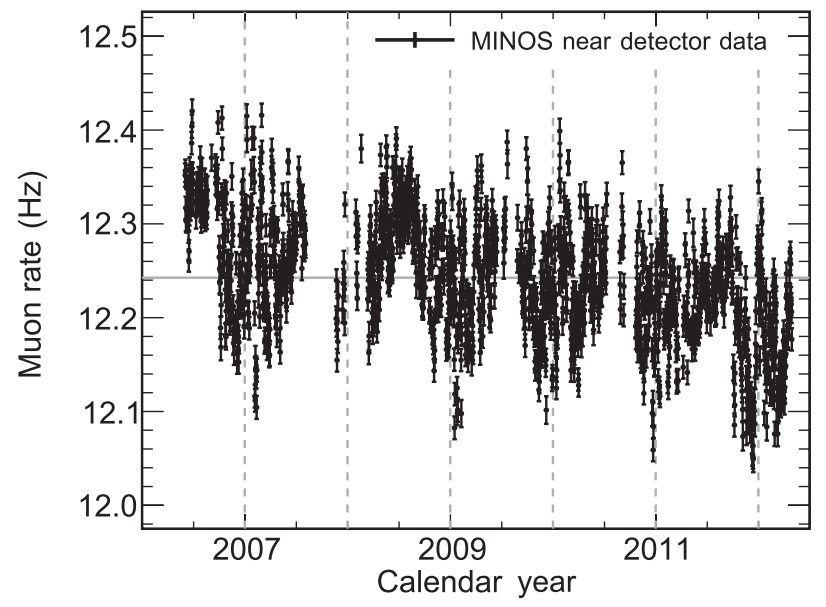

FIG. 4. The observed muon rate at the MINOS ND as a function of time. Each data point corresponds to one day of data. The horizontal line is the detector average of $12.2458 \mathrm{~Hz}$. The dashed vertical lines mark the start of new calendar years. 


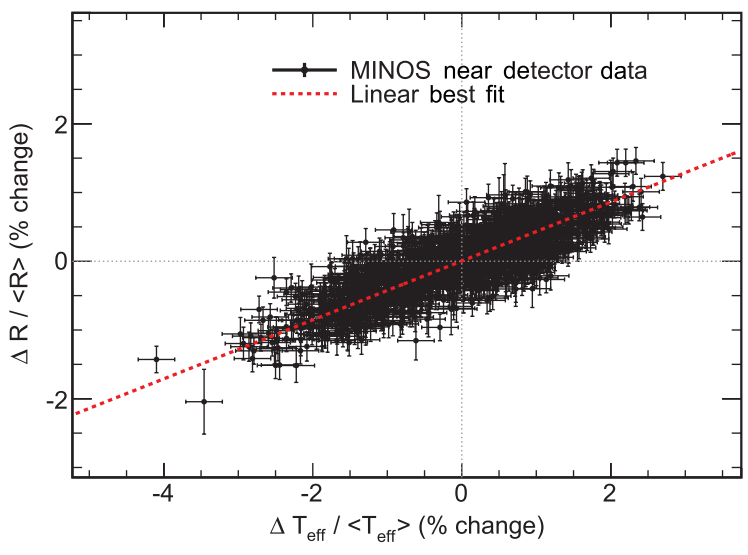

FIG. 5 (color online). Distribution of $\Delta R /\langle R\rangle$ versus $\Delta T_{\text {eff }} /\left\langle T_{\text {eff }}\right\rangle$. Each data point corresponds to approximately six hours of MINOS ND data. The $y$-axis uncertainty is purely statistical. The $x$-axis uncertainty is $0.2 \%$ and is the point-to-point variation in the ECMWF data. The best fit slope, equivalent to $\alpha_{T}$, is $0.465 \pm 0.003$ (stat.). To reduce clutter, only every fifth data point is shown.

of $183.4 \pm 0.3$ days. As expected the modulation periods for both data sets are close to one year with the maxima occurring in the summer months.

Figure 5 shows the percentage change in the observed muon rate $\Delta R /\langle R\rangle$ versus the percent change in effective temperature $\Delta T_{\text {eff }} /\left\langle T_{\text {eff }}\right\rangle$. The two data sets are strongly correlated with a correlation coefficient $\rho=0.81$. The best fit value for $\alpha_{T}$ is $0.465 \pm 0.003$ (stat.).

The data in Fig. 4 indicate that the mean muon rate has decreased over the lifetime of the experiment. The source of this small but apparently steady decrease has not been conclusively identified. Three possible sources of this rate loss have been identified: (i) solar cycle effects on the primary cosmic ray rate, (ii) secular variations in the local magnetic field and (iii) detector degradation effects. Since the effect seems larger for longer tracks than for shorter tracks, a detector degradation explanation is disfavored. The rate loss could possibly be reflected in the temperature and represent a shortcoming of the temperature data. Biases and trends have been reported with ERA-Interim temperature data, most notably around 200-100 $\mathrm{hPa}$ [23]. These have been attributed to warm biases in aircraft observations entering the data assimilation. However, these can only explain $10 \%$ of the observed rate loss, as comparative temperature biases with Radiosonde data are $0.1 \mathrm{~K}$ [24]. Regardless of its causes, the effect can be almost entirely removed by assuming a linear decline and refitting the data to obtain $\alpha_{T}$. To do this, Eq. (1) can be modified to account for a rate loss by redefining $\left\langle R_{\mu}\right\rangle$ as

$$
\left\langle R_{\mu}^{t}\right\rangle=\left\langle R_{\mu}^{0}\right\rangle \cdot\left(1-f \cdot \frac{t}{365.25}\right)
$$

where $f$ is the fractional loss rate, $t$ is the number of days since January 1, 2010 and $\left\langle R_{\mu}^{0}\right\rangle$ is the mean muon rate on that date. The data were again fit, this time allowing for the mean muon rate to change as a function of time according to Eq. (6), and the best fit value of $\alpha_{T}$ was calculated to be $0.428 \pm 0.003$ (stat.). This value comprises our result using the standard definition of effective temperature.

\section{B. Systematic uncertainties}

The systematic uncertainties on $\alpha_{T}$ can be loosely grouped into two sources, those derived from the analysis of the muon data and those relating to the calculation of the effective temperature. This section elaborates on the determination of these uncertainties whose magnitudes are given in Table I.

The nominal effective temperature has been determined using the atmospheric temperature profile directly above the detector. However, the temperature profile will change as a function of latitude and longitude. This implies that the effective temperature, and therefore $\alpha_{T}$, is a function of the arrival direction of the muon. The muon data were separated into northerly and southerly going components, in order to maximize exposure to differences in the atmospheric temperature profiles. A value of $\alpha_{T}$ (using the nominal $T_{\text {eff }}$ ) was calculated for each data set. The maximum difference from the nominal value, \pm 0.017 , is the systematic uncertainty due to the variability in the temperature profile.

The muon rate is clearly decreasing a small amount since the beginning of the experiment, but the decrease need not be linear as our fit assumes. The systematic uncertainty associated with decreasing event rate, based upon the change implied by allowance for the fitted rate loss, is estimated to be \pm 0.018 .

For this analysis the two integrals in the definition of $\mathrm{T}_{\text {eff }}$ in Eq. (2) were evaluated using a quadratic interpolation technique. Multiple integration techniques were tested and the maximum difference from the employed method, \pm 0.023 , is the systematic uncertainty associated with the integration technique. To evaluate the uncertainty

TABLE I. The systematic uncertainties associated with the nominal measurement of $\alpha_{T}$.

\begin{tabular}{lc}
\hline \hline Systematic & $\alpha_{T}$ uncertainty in $\alpha_{T}$ \\
\hline Muon direction & 0.017 \\
Rate loss fit & 0.018 \\
Integration & 0.023 \\
ECMWF model & 0.018 \\
Temperature series & 0.045 \\
& \\
$\left\langle\mathrm{E}_{\text {th }} \cos \theta\right\rangle$ & $\mathrm{T}_{\text {eff }}$ calculation \\
Net systematic & \\
\hline \hline
\end{tabular}




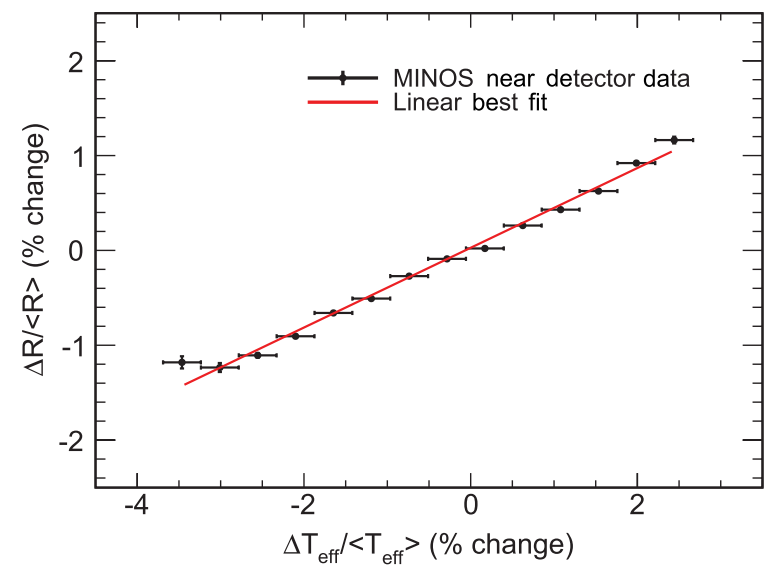

FIG. 6 (color online). The change in the observed muon rate versus the change in the effective temperature. In this figure the muon rate has been calculated as a function of effective temperature rather than on a point-to-point basis as in Fig. 5 .

associated with the ECMWF temperature data themselves, the $\alpha_{T}$ parameter was re-evaluated using an older 21 pressure-level ECMWF model. Fitting only the data from the periods where the two models overlap, the best fit values are found to differ by \pm 0.018 . This difference is taken to be the uncertainty due to the ECMWF model.

The nominal value of $\alpha_{T}$ given in Sec. III A was determined by comparing the muon rate measured over a six hour interval to the average effective temperature for that period. An alternative approach would be to calculate the mean muon rate for a given effective atmospheric temperature. The data have been grouped into $1 \mathrm{~K}$ bins in temperature (roughly twice the statistical uncertainty) and the muon rate determined as the total number of events divided by the total time for the data points that occur in that bin. Figure 6 shows the percent change in mean muon rate versus the percent change in effective atmospheric temperature. The best fit value for $\alpha_{T}$ is $0.420 \pm 0.015$ (stat.). The deviation of this value from the nominal value, \pm 0.045 , is the systematic uncertainty associated with the analysis technique.

Last, there is uncertainty in the parameters used to calculate the effective temperature. Of the parameters studied in [12], namely $\mathrm{R}_{K / \pi}, \epsilon_{K}, \epsilon_{\pi}, \gamma$ and $\left\langle\mathrm{E}_{\text {th }} \cos \theta\right\rangle$, it was found that only $\left\langle\mathrm{E}_{\mathrm{th}} \cos \theta\right\rangle=(54 \mathrm{GeV} \pm 10 \%)$ had a non-negligible impact, \pm 0.0024 , on the calculated value of $\alpha_{T}$.

In summary, the effective temperature coefficient $\alpha_{T}$ at the MINOS ND is determined to be $0.428 \pm 0.003$ (stat.) \pm 0.059 (syst.).

\section{Theoretical prediction}

The theoretical value of $\alpha_{T}$ can be expressed in terms of the differential muon intensity $\mathrm{I}_{\mu}$ as [1]

$$
\alpha_{T}=-\frac{E_{\mathrm{th}}}{I_{\mu}^{0}} \frac{\partial I_{\mu}}{\partial E_{\mathrm{th}}}-\gamma .
$$

Performing the differentiation yields $[1,13]$

$$
\alpha_{T}=\frac{1}{D_{\pi}} \frac{1 / \epsilon_{K}+A_{K}^{1}\left(D_{\pi} / D_{K}\right)^{2} / \epsilon_{\pi}}{1 / \epsilon_{K}+A_{K}^{1}\left(D_{\pi} / D_{K}\right) / \epsilon_{\pi}}-\delta
$$

where

$$
D_{\pi / K}=\frac{\gamma}{\gamma+1} \frac{\epsilon_{\pi / K}}{1.1\left\langle E_{\mathrm{th}} \cos \theta\right\rangle}+1
$$

and the correction for muon decay $\delta$ is

$$
\delta=1.0336 \frac{\gamma}{\gamma+1} \ln \left(\frac{8.5833}{\cos \theta}\right) \frac{1}{\left\langle E_{\mathrm{th}} \cos \theta\right\rangle} .
$$

A Monte Carlo simulation was used to determine the theoretically expected value of $\alpha_{T}$. A muon energy and $\cos \theta$ were chosen randomly from the differential muon intensity spectrum [25]. The muon was then randomly assigned an azimuthal angle $\phi$. The threshold energy for a particular direction in $\theta$ and $\phi$ was determined using the MINOS overburden; details are given in Ref. [18]. The $\alpha_{T}$ parameter was calculated using Eq. (8). This process was repeated to obtain an $\alpha_{T}$ distribution generated from 10,000 successful muon events. The theoretical value of $\alpha_{T}$ is the mean of this distribution and is equal to $0.390 \pm 0.004$ (stat.). The theoretical value of $\alpha_{T}$ has a systematic uncertainty due to the uncertainties in the parameters used to evaluate Eq. (8). Table II gives the $\pm 1 \sigma$ uncertainties with the respective parameters.

The measured value of $\alpha_{T}^{\exp }=0.428 \pm 0.003$ (stat.) \pm 0.059 (syst.) is larger than, but consistent with, the theoretical prediction of $\alpha_{T}^{\text {theory }}=0.390 \pm 0.004$ (stat.) \pm 0.028 (syst.).

\section{ZENITH ANGLE ANALYSIS}

The measurement of $\alpha_{T}$ in the preceding section assumes that the variation in the muon rates at all zenith angles only depends upon the vertical effective temperature [Eq. (2)]. However, cosmic ray primaries with large zenith angles

TABLE II. The $\pm 1 \sigma$ systematic uncertainties on the theoretical value of $\alpha_{T}$ at the MINOS ND.

\begin{tabular}{lc}
\hline \hline Systematic & Uncertainty on $\alpha_{T}$ \\
\hline $\mathrm{R}_{K / \pi}=0.149 \pm 0.06$ & 0.011 \\
$\epsilon_{K}=0.850 \pm 0.014 \mathrm{TeV}$ & 0.00016 \\
$\epsilon_{\pi}=0.115 \pm 0.003 \mathrm{TeV}$ & 0.00567 \\
$\gamma=1.7 \pm 0.1$ & 0.00556 \\
$\left\langle E_{\mathrm{th}} \cos \theta\right\rangle=54 \mathrm{GeV} \pm 10 \%$ & 0.0243 \\
Net systematic & \pm 0.028 \\
\hline \hline
\end{tabular}




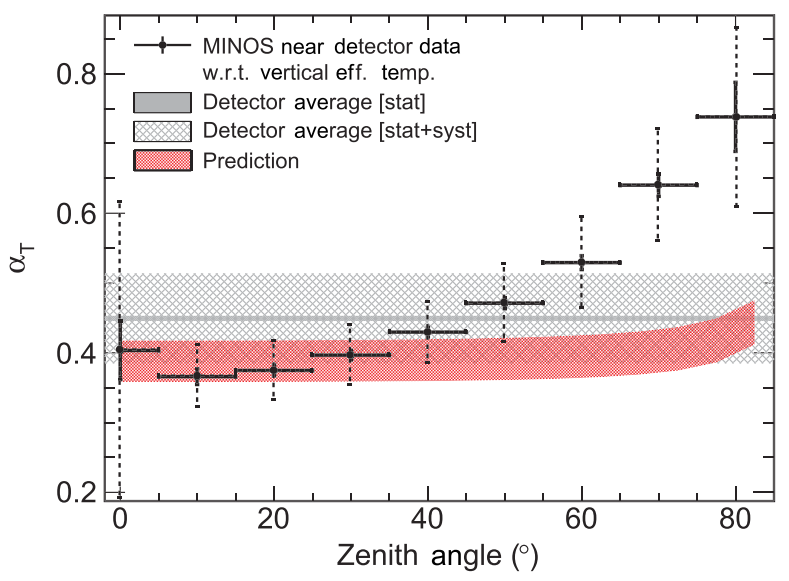

FIG. 7 (color online). The parameter $\alpha_{T}$ as a function of zenith angle when $T_{\text {eff }}$ is calculated using Eq. (2). The inner error bars on the data points are statistical; the outer error bars include both statistical and systematic uncertainties. The gray band is the detector average, and the red band is the theoretical prediction. The systematic difference of the data from the average suggests that the vertical effective temperature is inadequate for data over a range of zenith angles.

interact higher in the atmosphere where the temperature fluctuations are larger. Consequently, the variation in the muon rates should increase as a function of the zenith angle and, with no redefinition of the effective temperature, the measured values of $\alpha_{T}$ should increase as well. In this section we will calculate $\alpha_{T}$ as a function of zenith angle using both the vertical and angular effective temperatures.

In addition to the selection criteria outlined in Sec. II A the angular resolution of the muon tracks is required to be better than $5^{\circ}$. So as to not change the underlying $\mathrm{E}_{\mathrm{th}} \cos \theta$

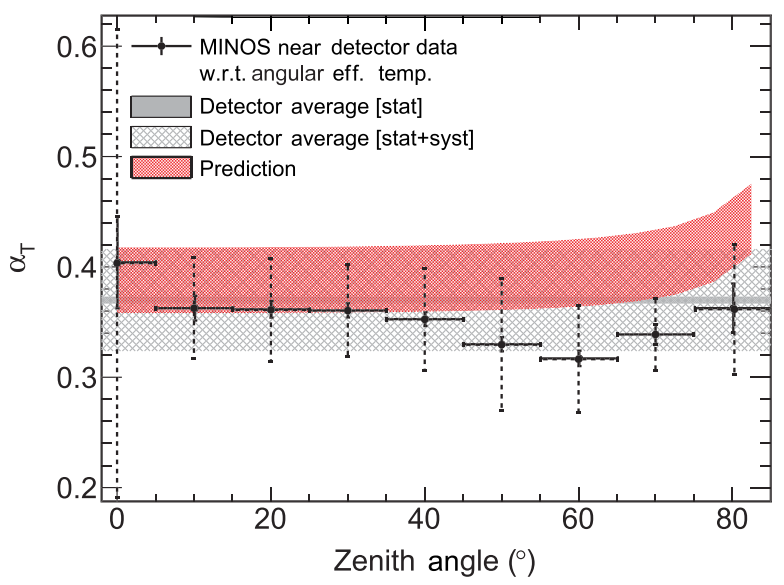

FIG. 8 (color online). The $\alpha_{T}$ parameter as a function of zenith angle when $T_{\text {eff }}(\theta)$ is calculated using Eq. (11). The inner error bars on the data points are statistical; the outer error bars include both statistical and systematic uncertainties. The gray band is the detector average calculated using $T_{\mathrm{eff}}^{\text {weight }}$ as defined in Eq. (12). The red band is the theoretical prediction and is the same as in Fig. 7.

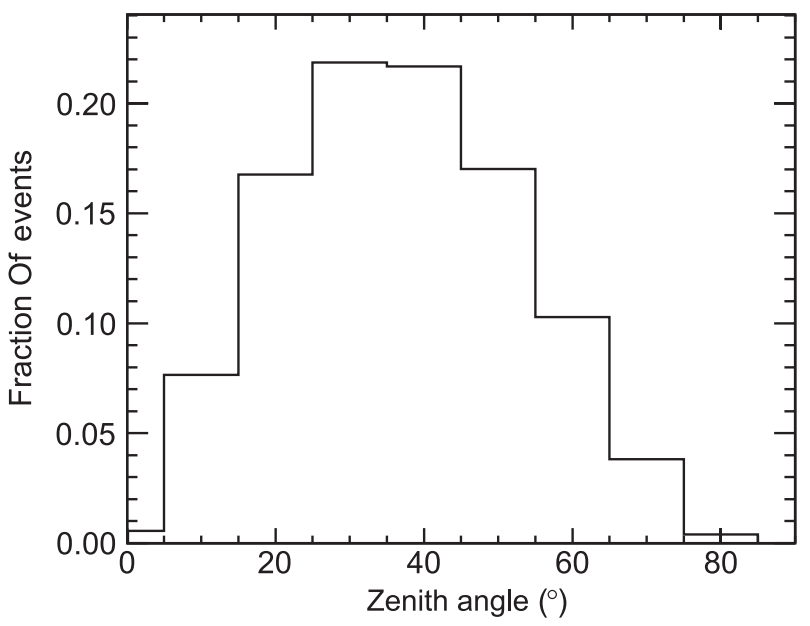

FIG. 9. Reconstructed zenith angle distribution for single muon events observed in the MINOS ND.

distribution of the muons, the changes in event selection were kept to a minimum. The value of $\alpha_{T}$ was determined for this resolution-enhanced data sample to be statistically consistent with the nominal value, 0.428 .

Figure 7 gives the measured $\alpha_{T}$ as a function of zenith angle when $T_{\text {eff }}$ is calculated using Eq. (2). The data are grouped into, and the values of $\alpha_{T}$ calculated for, nine zenith angle bins. The first bin is from $0-5^{\circ}$, and the remaining eight bins each cover the next $10^{\circ}$ increments. The theoretical prediction as a function of zenith angle is calculated using the Monte Carlo method outlined in Sec. III C but averaged instead over the zenith angle bins. It should be noted that the theoretical value of $\alpha_{T}$ is independent of the atmospheric temperature and is therefore not affected by our zenith angle corrections. Not surprisingly the measured value of $\alpha_{T}$ increases with zenith angle and does so more rapidly than the theoretical prediction.

Equation (2) was modified to account for the increased height of the primary cosmic ray interaction at larger zenith angles. The angular effective temperature for a particular zenith angle $\theta$ is simply

TABLE III. The systematic uncertainties associated with the measurement of $\alpha_{T}^{\text {weight }}$.

\begin{tabular}{lc}
\hline \hline Systematic & Uncertainty on $\alpha_{T}^{\text {weight }}$ \\
\hline Muon direction & 0.020 \\
Rate loss fit & 0.017 \\
Integration & 0.033 \\
ECMWF model & 0.014 \\
Temperature series & 0.011 \\
& $\mathrm{~T}_{\text {eff }}$ calculation \\
$\mathrm{R}_{K / \pi}$ & \\
$\left\langle E_{\text {th }} \cos \theta\right\rangle$ & 0.00186 \\
Net $\operatorname{systematic}$ & 0.0036 \\
\hline \hline
\end{tabular}




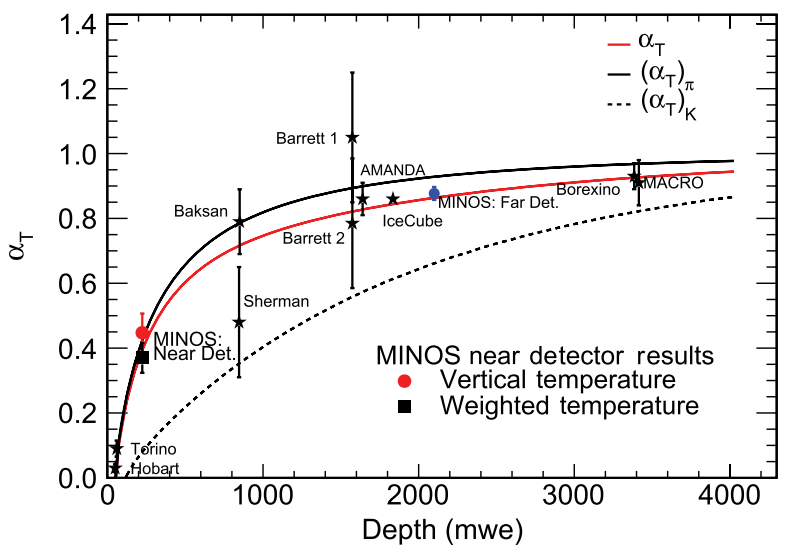

FIG. 10 (color online). The parameter $\alpha_{T}$ as a function of detector depth. The two measurements of this analysis are shown in black. The middle red line is the theoretical prediction, Eq. (8), for a detector at depth in $\left(\rho=2.65 \mathrm{gm} / \mathrm{cm}^{3}\right)$ standard rock. The top (bottom) line is the expected value of $\alpha_{T}$ assuming a pion-only (kaon-only) model determined by setting $A_{K}^{1}=$ $0\left(A_{K}^{1} \rightarrow \infty\right)[20]$.

$$
T_{\text {eff }}=\frac{\int_{0}^{\infty} d(X / \cos \theta) T(X) W(X)}{\int_{0}^{\infty} d(X / \cos \theta) W(X)} .
$$

The formulas for the weights $W(X)$ are unchanged from Eq. (3); only the depth $(X \rightarrow X / \cos \theta)$ and $E_{\mathrm{th}} \cos \theta$ arguments change with zenith angle. The $1 / \cos \theta$ terms in the denominator and numerator do cancel but have been left in for completeness. Figure 8 shows $\alpha_{T}$ as a function of zenith angle when the effective temperature $T_{\text {eff }}(\theta)$ has been calculated using Eq. (11). The figure shows that the values of $\alpha_{T}$ calculated in this manner are now consistent with the theoretical prediction.

To determine a single value of $\alpha_{T}$ for the MINOS ND, a single measure of temperature is initially defined using Eq. (2), as a weighted average based upon the observed muon angular distribution. The weighted angular effective temperature is then defined as

$$
T_{\mathrm{eff}}^{\mathrm{weight}}=\sum_{i=1}^{M} F_{i} \cdot T_{\mathrm{eff}}\left(\theta_{i}\right)
$$

where $M$ is the number of zenith angle bins. $T_{\text {eff }}\left(\theta_{i}\right)$ is the angular effective temperature in bin $i . F_{i}$ is the fraction of muons occurring in that bin, the distribution of which is shown in Fig. 9.

Using the weighted effective temperature defined in Eq. (12), and repeating the data analysis and systematic calculations as described in Sec. III, the weighted effective temperature coefficient $\alpha_{T}^{\text {weight }}$ at the MINOS ND is found to be

$$
\alpha_{T}^{\text {weight }}=0.352 \pm 0.003 \text { (stat.) } \pm 0.046 \text { (syst.). }
$$

The magnitudes of the individual systematic uncertainties are given in Table III. This result is consistent with the theoretical prediction of $\alpha_{T}^{\text {theory }}=0.390 \pm 0.004$ (stat.) \pm 0.028 (syst.).

Figure 10 shows the new MINOS ND results and all the known measured values of $\alpha_{T}$ as a function of detector depth. The figure includes results from Barrett 1,2 [1], AMANDA [7], ICECUBE [10], MACRO [6], Torino [3], Hobart [4], Sherman [2], Baksan [5], Borexino [8] and the MINOS FD [12]. The data are fully consistent with the prediction that $\alpha_{T}$ increases with detector depth (equivalent to increasing values of $E_{\mathrm{th}} \cos \theta$ ) and asymptotically approaches unity for very large detector depths.

\section{CONCLUSION}

A measurement of the effective temperature coefficient $\alpha_{T}$ has been performed using nearly six years of MINOS ND data. The value of this coefficient is determined to be

$$
\alpha_{T}=0.428 \pm 0.003 \text { (stat.) } \pm 0.059 \text { (syst.). }
$$

Additionally, a method that improves upon the conventional approach to determination of $\alpha_{T}$ using an underground detector of large angular acceptance has been demonstrated in this work. The improvement is achieved by accounting for the variance in the modulation of muon rate as a function of zenith angle. A weighting of the effective temperature as a function of zenith angle based on the relative flux of muons improves consistency and gives

$$
\alpha_{T}^{\text {weight }}=0.352 \pm 0.003 \text { (stat.) } \pm 0.046 \text { (syst.). }
$$

The zenith angle acceptance of an underground detector depends on both the geometry of the detector and the geometry of the overburden. The correction for zenith angle in the determination of $\alpha_{T}$ is relatively more important for detectors which have a vertically concave overburden, since these experience higher fluxes at large zenith angles. However the zenith angle correction is also important for detectors at depths less than 1000 mwe where $\alpha_{T}$ is rapidly changing, as shown in Fig. 10.

\section{ACKNOWLEDGMENTS}

This work was supported by the U.S. DOE, the United Kingdom STFC, the U.S. NSF, the state of Minnesota and University of Minnesota, and Brazil's FAPESP, CNPq and CAPES. We are grateful to the personnel of Fermilab for their contributions to the experiment. 
[1] P. Barrett, L. Bollinger, G. Cocconi, Y. Eisenberg, and K. Greisen, Rev. Mod. Phys. 24, 133 (1952).

[2] N. Sherman, Phys. Rev. 93, 208 (1954).

[3] G. C. Castagnoli and M. Dodero, J. Geophys. Res. 74, 2414 (1969).

[4] A. Fenton, R. Jacklyn, and R. Taylor, Nuovo Cimento 22, 285 (1961).

[5] Y. Andreyev et al. (Baksan Collaboration), in Proceedings of the 20th ICRC [3, 270 (1987)].

[6] M. Ambrosio et al. (MACRO Collaboration), Astropart. Phys. 7, 109 (1997).

[7] A. Bouchta (AMANDA Collaboration), in Proceedings of the 26th ICRC [2, 108 (1999)].

[8] G. Bellini et al. (Borexino Collaboration), J. Cosmol. Astropart. Phys. 05 (2012) 015.

[9] M. Selvi (LVD Collaboration), in Proceedings of the 31st ICRC, Lodz, Poland, 2009.

[10] P. Desiati et al. (IceCube Collaboration), Proceedings of the 32nd ICRC, Beijing, China, 2011.

[11] S. Osprey et al. (MINOS Collaboration), Geophys. Res. Lett. 36, L05809 (2009).

[12] P. Adamson et al. (MINOS Collaboration), Phys. Rev. D 81, 012001 (2010).

[13] E. W. Grashorn J. K. de Jong, M. C. Goodman, A. Habig, M. L. Marshak, S. Mufson, S. Osprey, and P. Schreiner, Astropart. Phys. 33, 140 (2010).
[14] D. P. Dee et al., Q. J. R. Meteorol. Soc. 137, 553 (2011).

[15] D. G. Michael et al. (MINOS Collaboration), Nucl. Instrum. Methods Phys. Res., Sect. A 596, 190 (2008).

[16] K. Anderson et al., Report No. FERMILAB-DESIGN1998, 1998.

[17] N. Tagg, A. De Santo, A. Weber, A. Cabrera, P. S. Miyagawa, M. A. Barker, K. Lang, D. Michael, R. Saakyan, and J. Thomas, Nucl. Instrum. Methods Phys. Res., Sect. A 539, 668 (2005).

[18] P. Adamson et al. (MINOS Collaboration), Phys. Rev. D 83, 032011 (2011).

[19] I. Durre, R. S. Vose, and D. B. Wuertz, J. Clim. 19, 53 (2006).

[20] E. W. Grashorn, Ph. D. thesis, University of Minnesota (Institution Report No. FERMILAB-THESIS-2008-06, 2008).

[21] S. Sagisaka, Nuovo Cimento 9C, 809 (1986).

[22] M. Motoki et al., Astropart. Phys. 19, 113 (2003).

[23] A. J. Simmons, P. Poli, D. P. Dee, P. Berrisford, H. Hersbach, S. Kobayashib, C. Peubey, Q. J. R. Meteorol. Soc. 140, 329 (2014).

[24] D. P. Dee and S. Uppala, Q. J. R. Meteorol. Soc. 135, 1830 (2009).

[25] T. K. Gaisser, Cosmic Rays and Particle Physics (Cambridge University Press, Cambridge, 1990). 